\section{sis} (2) (1)

\section{OPEN ACCESS}

- Additional material is published online only. To view please visit the journal online (http://dx.doi.org/10.1136/ thoraxinl-2014-205278)

${ }^{1}$ Department of Respiratory Medicine, Barts Health NHS Trust, London, UK

${ }^{2}$ Department of Medicines Management and Pharmacy Services, Leeds University Hospitals Trust, Leeds, UK ${ }^{3}$ Department of Respiratory Medicine, The Whittington Hospital, London, UK ${ }^{4}$ Department of Respiratory Medicine, Queen Alexandra Hospital, Portsmouth, UK ${ }^{5}$ Imperial College London, Chest and Allergy Clinic, London, UK

\section{Correspondence to} Dr Jessica Louise Potter, Department of Respiratory Medicine, London Chest Hospital, Bonner Road, London E2 9JX, UK Jessica.keal@gmail.com, lessica.potter@ bartshealth.nhs.uk

Received 13 February 2014 Revised 29 April 2014 Accepted 21 May 2014 Published Online First 13 June 2014

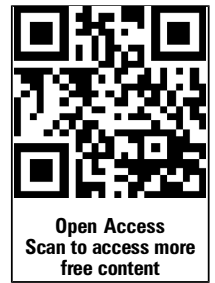

CrossMark

\section{To cite: Potter JL,} Capstick T, Ricketts WM, et al. Thorax 2015;70: 297-298.

\title{
A UK-based resource to support the monitoring and safe use of anti-TB drugs and second-line treatment of multidrug-resistant TB
}

\author{
J L Potter, ${ }^{1}$ T Capstick, ${ }^{2}$ W M Ricketts, ${ }^{3}$ N Whitehead, ${ }^{4}$ O M Kon ${ }^{5}$
}

\section{ABSTRACT}

Using the best available evidence and expert consensus, this document provides guidance for adverse effect monitoring in multidrug-resistant TB (MDR-TB). It includes recommendations for baseline tests, routine drug and toxicity monitoring guides as well as individual drug monographs for all drugs currently available in the UK to treat TB. These recommendations provide a structure through which healthcare professionals can better manage the complex drug regimens required for the treatment of MDR-TB; minimising the risk of adverse incidents and helping to improve patients' tolerance, compliance and treatment completion.

\section{CLINICAL CONTEXT}

Multidrug-resistant TB (MDR-TB) is caused by bacteria that do not respond to the two most powerful anti-TB drugs; isoniazid and rifampicin. MDR-TB is a growing concern; the $\mathrm{WHO}$ estimates that there were 450000 new cases of MDR-TB in 2012 and global incidence almost doubled in that same year. In the UK, MDR-TB cases have nearly tripled over the last decade and we continue to discover cases of $\mathrm{XDR} \mathrm{TB}^{2}$; defined as TB that is resistant to both isoniazid and rifampicin (MDR-TB) plus a fluoroquinolone and at least one of the injectable agents (amikacin, capreomycin, kanamycin).

The cost of treatment for MDR-TB is estimated to be 10 times that of fully sensitive $\mathrm{TB}^{3}$ Treatment regimens are complex and prolonged with a high risk of serious adverse drug reactions (ADRs). Treatment is associated with significant morbidity and mortality, threatening adherence ${ }^{4}$ and increasing the risk of transmission of these difficult to treat strains of TB. The Global plan to Stop TB aims for successful treatment completion in $75 \%$ for all patients with MDR-TB; however, completion rates for MDR-TB in the UK were below this target at only $70.6 \%$ for the period between 2004 and 2007.

We are not aware of any previous guidelines in the UK that have adequately advised clinicians on the baseline testing that should be performed and how to ensure that anti-TB drugs are used safely and effectively in patients treated for drug-resistant TB. We would like to introduce the first UK guidance for adverse-effect monitoring in MDR-TB.

\section{TARGET AUDIENCE}

The guidance is aimed at all healthcare workers in the field of TB and in particular those who work with MDR-TB. It is relevant not only to UK practitioners but those working with MDR-TB around the world. This guideline is to aid monitoring for adverse effects during the treatment of MDR-TB. It is not a treatment guide or a guide for monitoring the progress of treatment. Treatment of MDR-TB is complex and users of this guidance should ensure all aspects of treatment are in consultation with local experts and published guidance.

\section{SCOPE OF THE GUIDANCE}

The guidance consists of two main sections. The first, 'Baseline and generic tests for adverse effects monitoring in patients being treated for MDR-TB', provides advice on the frequency of monitoring that should occur at minimum in all patients on MDR-TB treatment (box 1).

The second section includes 'drug monographs' for all drugs currently used to treat MDR-TB, including amikacin, bedaquiline, capreomycin, clarithromycin, clofazamine, co-amoxiclav, cycloserine, ethambutol, imipenem-cilastatin, isoniazid, levofloxacin, linezolid, meropenem, moxifloxacin, ofloxacin, P-aminosalicylic acid, prothionamide, pyrazinamide, rifabutin, rifampicin, streptomycin and thioacetazone. Each monograph was based upon a standard layout as is shown in box 2 .

\section{METHODOLOGY}

This document was written using the best available published evidence and, where this was limited, expert consensus. The guideline team consists of five health professionals with experience treating patients with TB and MDR-TB; a TB pharmacist, a TB consultant physician, two respiratory registrars and a TB nurse.

An initial review of published guidelines was undertaken, which allowed us to identify gaps in

\section{Box 1 Baseline and routine monitoring}

\section{recommendations}

Baseline tests and ongoing monitoring recommendations for adverse effects monitoring

- Blood tests

- Audiology

- Visual acuity and colour discrimination testing

- ECG 


\section{Box 2 Standard layout of each 'drug monograph'}

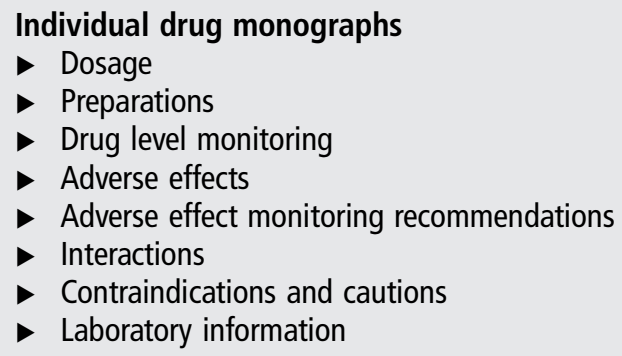

knowledge and to gain an overview of current practice. To produce the individual drug monographs, a two-step approach was taken. Tertiary reference sources, published reviews and international guidelines were analysed to provide the basis for individual drug monographs since these have collated data from robust clinical and pharmacokinetic studies. This was supported and enhanced by a literature review using Medline and hand searching of reference lists from published studies. The latter strategy was particularly important where there was a paucity of published data to support recommendations. One individual reviewed the abstracts from each search to identify potentially relevant studies. On occasion systematic reviews suggested a dosing range rather than specific doses, and in this situation, expert consensus was used to guide our dose recommendations. Where there was no clear evidence, current practice, our own experience and expert consensus were used.

Our multidisciplinary guideline development group held regular teleconferences and corresponded by email. When evidence was sparse, expert consensus was sought from the British Thoracic Society TB Special Advisory Group (SAG) and UK MDR-TB Advisory Service. When other specialty input was required, this was sought from experts in that field. Once the guideline was developed, it was submitted to the TB SAG for peer review. Feedback was reviewed by the committee and accepted or rejected based on supporting evidence and/or expert consensus.

\section{ONLINE ACCESS AND FUTURE DEVELOPMENTS}

This guidance is designed to be available as an online resource so that it can be updated when the need arises, such as when new drugs are launched or when new data that affect the use of existing anti-TB drugs become available. The guidance has been published online at http://www.tbdrugmonographs.co.uk and will be freely accessible to all.

In addition to this web version, software is being developed to facilitate the ease of use of the guidance. This application will enable the monitoring guidance to be customised for individual patients based on their specific drug regimen. This will also be made available through the website.
New literature will be reviewed annually and incorporated where relevant. Additionally, an open invitation for feedback is incorporated into the website and within the guidance document. Any feedback received will be discussed among the guideline team with any required changes incorporated into the relevant monographs.

\section{AUDIT AND RESEARCH RECOMMENDATIONS}

Audit of this guidance is suggested after it has been implemented in a centre for a reasonable period of time; we suggest 6-12 months. Results will be used to revise recommendations and assess impact on treatment outcomes, costs and patient experience.

\section{CONCLUSIONS}

We hope that by introducing a guideline to aid ADR monitoring in MDR-TB treatment we can improve treatment adherence, morbidity and mortality and reduce treatment costs.

Contributors JLP is responsible for the inception of this piece of work, wrote the paper and played a major role in writing the main guidance. TC did the main literature review and provided content for the main guidance. WMR provided expert advice and editorial assistance on the main document. NW provided advice and perspective in her role as an experience TB nurse. OMK provided expert advice and additional literature review for the main guidance and the paper. He coordinated and chaired the committee meetings throughout the development of the guidance. $\mathrm{He}$ is responsible for the content of the paper and the guidance and acts as guarantor for both.

Funding The publication of this work was funded by the NIHR Imperial Biomedical Research Council.

Competing interests JLP owns the web domain http://www.tbdrugmonographs. co.uk, but has no pecuniary interest in its use.

Provenance and peer review Not commissioned; externally peer reviewed.

Data sharing statement Feedback documents following consultation with the BTS multidrug-resistant special advisory group with our own responses to the feedback following a committee meeting are available. All meeting minutes are also available for review.

Open Access This is an Open Access article distributed in accordance with the Creative Commons Attribution Non Commercial (CC BY-NC 3.0) license, which permits others to distribute, remix, adapt, build upon this work non-commercially, and license their derivative works on different terms, provided the original work is properly cited and the use is non-commercial. See: http://creativecommons.org/ licenses/by-nc/3.0/

\section{REFERENCES}

1 World Health Organisation. Global Tuberculosis Report 2013. Geneva, Switzerland: World Health Organisation, 2013.

2 Public Health England. Tuberculosis in the UK-2013 report. Public Health England, 2013.

3 White VL, Moore-Gillon J. Resource implications of patients with multidrug resistant tuberculosis. Thorax 2000;55:962-3.

4 LJ P. Patterns of treatment interruption among patients with multidrug-resistant TB (MDR TB) and association with interim and final treatment outcomes. PLOS ONE 2013;8:e70064.

5 Anderson LF, Tamne S, Watson JP, et al. Treatment outcome of multi-drug resistant tuberculosis in the United Kingdom: retrospective-prospective cohort study from 2004 to 2007. Euro Surveill 2013;18:pii=20601. 